Pacific

Journal of

Mathematics

CONSTRUCTION DE FAMILLES MINIMALES DE COURBES GAUCHES

R. Hartshorne, M. Martin-Deschamps, and D. Perrin 


\title{
CONSTRUCTION DE FAMILLES MINIMALES DE COURBES GAUCHES
}

\author{
R. Hartshorne, M. Martin-Deschamps, and D. Perrin
}

Let $A$ be a noetherian local ring, and let $C$ in $\mathbb{P}_{A}^{3}$ be a family of curves, flat over $A$. We showed in an earlier paper how to associate to $C$ a locally free sheaf $N$ on $\mathbb{P}_{A}^{3}$, and we showed that two families of curves $C, C^{\prime}$ are in the same biliaison class if and only if the corresponding sheaves $N, N^{\prime}$ are pseudoisomorphic (generalization of the theorem Rao). In this paper we show how to find all the flat families of curves $C$ associated to a given locally free sheaf $N$ and its twists, starting with the minimal family $C_{0}$. We show also that all other families are obtained from the minimal family by a sequence of elementary biliaisons and a deformation (generalization of the theorem of Lazarsfeld Rao). The calculations are algorithmic in terms of a presentation of $N$.

\section{Introduction.}

Soit $A$ un anneau local noethérien, $t$ le point fermé de $T=\operatorname{Spec} A$ et soit $\mathbf{P}_{A}^{3}$ l'espace projectif associé. Si $\mathcal{C}$ est une famille plate de courbes de $\mathbf{P}_{A}^{3}$, il existe une résolution de $\mathcal{C}$, dite de type $N$, c'est-à-dire une suite exacte $0 \rightarrow \mathcal{P} \rightarrow \mathcal{N} \rightarrow \mathcal{J}_{\mathcal{C}} \rightarrow 0$ avec $\mathcal{N}$ localement libre et $\mathcal{P}$ dissocié (somme directe de faisceaux inversibles) et le faisceau $\mathcal{N}$ est bien déterminé par $\mathcal{C}$ à pseudo-isomorphisme près (cf. [HMDP1]), de sorte qu'on a une application $\Phi$ qui à une courbe $\mathcal{C}$ associe la classe de pseudo-isomorphisme du faisceau $\mathcal{N}$. Dans [HMDP1] nous avons déterminé les fibres de $\Phi$ à décalage près en montrant que les faisceaux $\mathcal{N}=\Phi(\mathcal{C})$ et $\mathcal{N}^{\prime}=\Phi\left(\mathcal{C}^{\prime}\right)$ sont pseudo-isomorphes à décalage près si et seulement si les courbes $\mathcal{C}$ et $\mathcal{C}^{\prime}$ sont dans la même classe de biliaison (c'est une généralisation du théorème de Rao, cf. $[\mathbf{R}]$ ).

Par ailleurs, il est facile de voir que $\Phi$ est surjective à décalage près (cf. 2.7 ci-dessous ou $[\mathbf{S} 1]$ ), c'est-à-dire que si $\mathcal{N}$ est un faisceau localement libre sur $\mathbf{P}_{A}^{3}$ il existe une famille de courbes (lisses et connexes) $\mathcal{C}$ de $\mathbf{P}_{A}^{3}$, plate sur $A$, et un entier $h$ tels que $\mathcal{C}$ admette une résolution de type $N$ de la forme: $0 \rightarrow \mathcal{P} \rightarrow \mathcal{N} \rightarrow \mathcal{J}_{\mathcal{C}}(h) \rightarrow 0$.

L'intérêt de ce type de résultat d'existence provient de l'étude du schéma de Hilbert $H_{d, g}$ des courbes de degré $d$ et genre $g$ donnés et notamment 
des spécialisations qu'on peut obtenir lorsqu'on fait varier la cohomologie. Une question essentielle est de montrer l'existence d'une famille de courbes, paramétrée par un anneau de valuation discrète $A$, dont le point spécial $C_{0}$ (resp. le point générique $C$ ) est une courbe à cohomologie prescrite. La méthode que nous proposons pour cela s'inspire de la construction des courbes à partir des modules de Rao inaugurée dans [MDP1] IV. Elle consiste, à partir d'un objet (appelé triade) qui généralise le module de Rao, cf. [HMDP3], à déterminer un faisceau $\mathcal{N}$ convenable puis à construire la famille de courbes avec une résolution de type $N$ comme ci-dessus.

Pour ce type de problèmes, le résultat de 2.7 est insuffisant, puisque, s'il assure l'existence d'une courbe de la classe de biliaison associée à $\mathcal{N}$, il ne permet pas de contrôler le décalage $h$ et donc d'affirmer que la famille construite a les degré et genre voulus. Dans cet article nous déterminons toutes les familles de courbes de la classe de biliaison associée à un faisceau localement libre $\mathcal{N}$ donné, à commencer par les familles minimales, i.e., celles pour lesquelles l'entier $h$ est minimal.

Pour cela nous introduisons ci-dessous, cf. 2.4, une fonction $q: \mathbf{Z} \rightarrow$ $\mathbf{N}$ et un entier $b_{0}$ associés à ce faisceau. Cette fonction est définie à la manière de [MDP1, MDP3, MDP4] comme une fonction $q$ du faisceau $\mathcal{N}_{t}=\mathcal{N} \otimes_{A} k(t)$, associée non pas au module des sections globales $H_{*}^{0} \mathcal{N}_{t}$ tout entier, mais au sous-module des sections qui proviennent de $H_{*}^{0} \mathcal{N}$, cf. $\S 1$. Les familles de courbes dont la résolution de type $N$ correspond à $\mathcal{N}$ sont alors données par le théorème suivant :

Théorème 2.6. Soit $\mathcal{N}$ un faisceau sur $\mathbf{P}_{A}^{3}$ localement libre de rang $r$ et non dissocié. Soit $\mathcal{P}=\bigoplus_{n \in \mathbf{Z}} \mathcal{O}_{\mathbf{P}_{A}}(-n)^{p(n)}$ un faisceau dissocié de rang $r-1$. Alors, les conditions suivantes sont équivalentes:

i) Pour u général dans $H=\operatorname{Hom}_{\mathcal{O}_{\mathbf{P}}}(\mathcal{P}, \mathcal{N})$, on a une suite exacte:

$$
0 \rightarrow \mathcal{P} \stackrel{u}{\longrightarrow} \mathcal{N} \rightarrow \mathcal{J}_{\mathcal{C}}(h) \rightarrow 0
$$

où $\mathcal{C}$ est une famille de courbes de $\mathbf{P}_{A}^{3}$, plate sur $A$.

ii) La fonction $p$ vérifie les conditions suivantes:

1) on a $p^{\sharp}(n) \leq q^{\sharp}(n)$ pour tout $n \in \mathbf{Z}$,

2) s'il existe $n \leq b_{0}$ tel que l'on ait $p^{\sharp}(n)=q^{\sharp}(n)$ on a un isomorphisme $\left(\mathcal{P}_{\leq n}\right)_{t} \simeq \mathcal{N}_{t, \leq n}$ (cf. 2.4).

La valeur minimale du décalage $h$ est égale à $\sum_{n \in \mathbf{Z}} n q(n)+\operatorname{deg} \mathcal{N} . \quad L a$ famille de courbes correspondant à ce décalage minimum est donnée par une résolution comme ci-dessus, avec $p=q$.

Les deux corollaires suivants décrivent les courbes d'une classe de biliaison donnée: 
Corollaire 2.9. Soit $\mathcal{N}_{0}$ un faisceau localement libre extraverti (i.e., tel que $H_{*}^{1} \mathcal{N}_{0}^{\vee}$ soit nul) minimal (i.e., sans facteur direct dissocié).

Posons $q=q_{\mathcal{N}_{0}}$ et $h_{0}=\sum_{n \in \mathbf{Z}} n q(n)+\operatorname{deg} \mathcal{N}_{0}$.

1) Il existe une famille de courbes $\mathcal{C}_{0}$ et une résolution $0 \rightarrow \mathcal{P}_{0} \stackrel{v}{\longrightarrow} \mathcal{N}_{0} \rightarrow$ $\mathcal{J}_{\mathcal{C}_{0}}\left(h_{0}\right) \rightarrow 0$ avec $\mathcal{P}_{0}$ dissocié.

2) Si $\mathcal{C}_{1}$ est une famille de courbes de la classe de biliaison de $\mathcal{C}_{0}$, elle admet une résolution $0 \rightarrow \mathcal{P} \rightarrow \mathcal{N}_{0} \oplus \mathcal{L} \rightarrow \mathcal{J}_{\mathcal{C}_{1}}(h) \rightarrow 0$ avec $\mathcal{L}$ dissocié et on $a h \geq h_{0}$. Si d et $g$ (resp. $d_{0}$ et $\left.g_{0}\right)$ sont respectivement le degré et le genre de $\mathcal{C}_{1}$ (resp. $\left.\mathcal{C}_{0}\right)$ on a $d \geq d_{0}$ et $g \geq g_{0}$.

3) Réciproquement, pour tout $h \geq h_{0}$, il existe une famille de courbes $\mathcal{C}_{1}$ avec une résolution comme ci-dessus.

4) Si de plus on a $h=h_{0}, \mathcal{C}_{0}$ et $\mathcal{C}_{1}$ sont jointes par une déformation à cohomologie uniforme (cf. 2.8 ci-dessous). On dit que $\mathcal{C}_{0}$ est une famille minimale de courbes.

Corollaire 2.10 (Propriété de Lazarsfeld-Rao). Soit $\mathcal{C}_{0}$ une famille minimale de courbes et $\mathcal{C}$ une famille de courbes de la classe de biliaison de $\mathcal{C}_{0}$. Alors, il existe un entier $m \geq 0$ et une suite de courbes $\mathcal{C}_{0}, \mathcal{C}_{1}, \ldots, \mathcal{C}_{m}$ telle que $\mathcal{C}_{i+1}$ s'obtienne à partir de $\mathcal{C}_{i}$ par une biliaison élémentaire (cf. [HMDP1]) et $\mathcal{C}$ à partir de $\mathcal{C}_{m}$ par une déformation à cohomologie uniforme.

Lorsque le faisceau $\mathcal{N}$ est écrit comme image d'un morphisme de faisceaux dissociés $s: \mathcal{L}_{2} \rightarrow \mathcal{L}_{1}$ tel que la flèche $L_{2} \rightarrow H_{*}^{0} \mathcal{N}$ induite par $s$ soit surjective et que le conoyau de $s$ soit localement libre (ce qui est possible pour tout faisceau localement libre sur $\mathbf{P}_{A}^{3}$ ), nous donnons un algorithme de calcul de la fonction $q$, entièrement effectif en termes de certains mineurs de $s_{t}$, (cf. 3.1). Nous donnons quelques exemples simples d'applications de cet algorithme. Pour d'autres exemples et des applications de ces techniques dans le cadre des triades, le lecteur se reportera à [HMDP3].

\section{Notations générales.}

Si $A$ est un anneau, on pose $T=\operatorname{Spec} A$ et on note $\mathbf{P}_{A}^{3}$ ou $\mathbf{P}_{T}^{3}$ l'espace projectif de dimension 3 sur $T$. On note $R_{A}$ l'anneau $A[X, Y, Z, T]$. Si $\mathcal{F}$ est un faisceau cohérent sur $\mathbf{P}_{A}^{3}$ on note $H^{i} \mathcal{F}$ le $A$-module $H^{i}\left(\mathbf{P}_{A}^{3}, \mathcal{F}\right)$ et on pose $H_{*}^{i} \mathcal{F}=\bigoplus_{n \in \mathbf{Z}} H^{i} \mathcal{F}(n)$.

Soit $\mathcal{N}$ un faisceau cohérent sur $\mathbf{P}_{A}^{3}$ et $t$ un point de $T$. On pose $\mathcal{N}_{t}=$ $\mathcal{N} \otimes_{A} k(t)$. Si $u: \mathcal{E} \rightarrow \mathcal{F}$ est un morphisme de faisceaux on pose $u_{t}=$ $u \otimes_{A} k(t)$.

Si $A$ est local, un faisceau sur $\mathbf{P}_{A}^{3}$ sera dit dissocié s'il est de la forme $\mathcal{F}=\bigoplus_{i=1}^{r} \mathcal{O}_{\mathbf{P}_{A}}\left(-n_{i}\right)$ où les $n_{i}$ sont des entiers. Si $t$ est le point fermé de Spec $A$ et si $\mathcal{N}$ est plat sur $A$ il résulte de $[\mathbf{H}] 3.10$ et 7.9 que $\mathcal{N}$ est dissocié si et seulement si $\mathcal{N}_{t}$ l'est. 
Si $L$ est un $R_{A}$-module gradué libre de type fini (resp. $\mathcal{L}$ un faisceau dissocié), on peut l'écrire sous la forme $L=\bigoplus_{n \in \mathbf{Z}} R_{A}(-n)^{l(n)}$ (resp. $\mathcal{L}=$ $\left.\bigoplus_{n \in \mathbf{Z}} \mathcal{O}_{\mathbf{P}_{A}}(-n)^{l(n)}\right)$ où $l$ est une fonction de $\mathbf{Z}$ dans $\mathbf{N}$, à support fini, appelée fonction caractéristique de $L$ (resp. $\mathcal{L})$. Le plus grand entier $n$ tel que $l(n)$ soit non nul est noté $\sup L(\operatorname{resp} . \sup \mathcal{L})$.

Si $q$ est une fonction de $\mathbf{Z}$ dans $\mathbf{Z}$ nulle pour $n \ll 0$, on pose $q^{\sharp}(n)=$ $\sum_{k \leq n} q(k)$. La donnée de $q$ équivaut à celle de $q^{\sharp}$ en vertu de la formule $q(n)=q^{\sharp}(n)-q^{\sharp}(n-1)$.

Si $M$ est un $R_{A}$-module gradué et $n$ un entier on note $M_{\leq n}$ le sousmodule gradué de $M$ engendré par ses éléments de degré $\leq n$. Si $L$ est libre de fonction caractéristique $l$ on a rang $L_{\leq n}=l^{\sharp}(n)$.

Sur un corps $k$, on appelle courbe un sous-schéma de $\mathbf{P}_{k}^{3}$ de dimension 1 , sans composante ponctuelle (immergée ou non), c'est-à-dire localement de Cohen-Macaulay.

Une famille de courbes gauches $\mathcal{C}$ sur $T$ est un sous-schéma fermé de $\mathbf{P}_{T}^{3}$, plat sur $T$, et dont les fibres sont des courbes au sens précédent.

\section{Fonction $q$ relative à un sous-module de sections.}

\section{a) Notations.}

Dans ce premier paragraphe on travaille sur un corps $k$ infini. On pose $\mathbf{P}^{3}=\mathbf{P}_{k}^{3}$ et $R=R_{k}$. Si $x$ est un point de $\mathbf{P}^{3}$ et $u: \mathcal{E} \rightarrow \mathcal{F}$ un morphisme de faisceaux cohérents on note $u(x)$ l'application linéaire de $\mathcal{E} \otimes_{\mathcal{O}_{\mathbf{P}}} k(x)$ dans $\mathcal{F} \otimes_{\mathcal{O}_{\mathbf{P}}} k(x)$ induite par $u$. Le rang de $u$ est par définition le rang de $u(\xi)$ où $\xi$ désigne le point générique de $\mathbf{P}^{3}$. Les notations suivantes seront fixées dans tout le paragraphe 1:

Notations 1.0. On considère un faisceau cohérent sans torsion $\mathcal{N} \operatorname{sur} \mathbf{P}^{3}$, on pose $N=H_{*}^{0} \mathcal{N}$ et on se donne un sous- $R$-module gradué $N^{\prime}$ de $N$. Si $n$ est un entier, on note $\sigma_{\mathcal{N}, N^{\prime}, n}$ ou simplement, s'il n'y a pas d'ambiguïté, $\sigma_{n}: N_{n}^{\prime} \otimes_{k} \mathcal{O}_{\mathbf{P}}(-n) \rightarrow \mathcal{N}$ le morphisme canonique. On note $\mathcal{N}_{N^{\prime}, \leq n}$ son image qui est aussi le sous-faisceau de $\mathcal{N}$ engendré par $N_{\leq n}^{\prime}$.

Définition 1.1. Avec les notations de 1.0 on définit la fonction $q_{\mathcal{N}, N^{\prime}}$ (ou simplement $q$ s'il n’y a pas d'ambiguïté) comme suit: $q^{\sharp}(n)$ est le plus grand entier $m$ tel qu'il existe un faisceau dissocié $\mathcal{P} \subset \mathcal{N}$, de rang $m$, vérifiant $H_{*}^{0} \mathcal{P} \subset N^{\prime}$ et $\sup \mathcal{P} \leq n$, tel que $\mathcal{N} / \mathcal{P}$ soit sans torsion.

Remarques 1.2. $\quad 0$ ) On notera que les entiers $m$ qui vérifient les conditions de 1.1 forment un intervalle $]-\infty, a]$.

1) Il est clair que $q^{\sharp}$ est une fonction croissante, donc que $q(n)=q^{\sharp}(n)-$ $q^{\sharp}(n-1)$ est $\geq 0$. De plus $q^{\sharp}$ est nulle pour $n \ll 0$. En effet, comme $\mathcal{N}$ est sans torsion, on a $H^{0} \mathcal{N}(n)=0$ pour $n \ll 0$ (cf. [HMDP1] 0.2). Enfin, comme $q^{\sharp}$ est bornée par le rang de $\mathcal{N}$ elle est constante pour $n \gg 0$. On en déduit que $q$ est à support fini. 
2) Par rapport à des résultats antérieurs (cf. [MDP1, MDP3]), la différence essentielle réside dans le fait que le faisceau $\mathcal{P}$ est "à valeurs dans $N^{\prime \prime \prime}$. Il y a essentiellement deux cas d'application de cette situation:

a) le cas banal, $N=N^{\prime}$, qui redonne la fonction $q$ ordinaire, voir $\S$ d) le calcul explicite de $q$,

b) le cas "à paramètres" qui fait l'objet du $\S 2$ : on part d'un faisceau $\mathcal{N}$ sur $\mathbf{P}_{A}^{3}$, où $A$ est un anneau local de corps résiduel $k(t)$, on considère le faisceau $\mathcal{N}_{t}=\mathcal{N} \otimes_{A} k(t)$ et on prend, comme sous-module $N^{\prime}$ de $H_{*}^{0} \mathcal{N}_{t}$ l'image canonique de $\left(H_{*}^{0} \mathcal{N}\right) \otimes_{A} k(t)$.

Proposition 1.3. On reprend les notations de 1.0. Soit $x \in \mathbf{P}^{3}$ un point de codimension $\leq 1$. On a, pour tout $n \in \mathbf{Z}, q^{\sharp}(n) \leq \operatorname{rang} \sigma_{n}(x)$.

Démonstration. Par définition de $q$ il existe une suite exacte $0 \rightarrow \mathcal{P} \stackrel{u}{\longrightarrow} \mathcal{N}$ $\rightarrow \mathcal{E} \rightarrow 0$ avec $\sup \mathcal{P} \leq n, \operatorname{rang} \mathcal{P}=q^{\sharp}(n), H_{*}^{0} \mathcal{P} \subset N^{\prime}$ et $\mathcal{E}$ sans torsion. Si $x$ est un point de codimension $\leq 1, \mathcal{E}$ est plat en $x$ de sorte que la suite reste exacte en tensorisant par $k(x)$. Comme $\mathcal{P}$ est en degrés $\leq n$ l'image de $u(x)$ est contenue dans celle de $\sigma_{n}(x)$ et on en déduit le résultat.

b) Le théorème principal.

Soit $H$ un $k$-espace vectoriel de dimension finie. Nous dirons qu'une propriété $P$ des éléments de $H$ est vraie pour $h$ "général" s'il existe un ouvert de Zariski non vide $U$ du schéma affine associé à $H$ tel que $P$ soit vraie pour tout $h \in U$. Puisque $k$ est infini, un tel ouvert a des points rationnels.

Lemme 1.4. On reprend les notations de 1.0. Soit $n \in \mathbf{Z}$. On pose $\sigma=\sigma_{n}$. Les assertions suivantes sont équivalentes:

1) Pour $s$ général dans $N_{n}^{\prime}$ on a une suite exacte $0 \rightarrow \mathcal{O}_{\mathbf{P}}(-n) \stackrel{s}{\longrightarrow} \mathcal{N} \rightarrow$ $\mathcal{E} \rightarrow 0$ avec $\mathcal{E}$ sans torsion.

2) On a les deux conditions suivantes:

a) Pour tout point $x$ de codimension 1 de $\mathbf{P}^{3}$, on a $\operatorname{rang} \sigma(x) \geq 1$,

b) on a - ou bien rang $\sigma \geq 2$,

- ou bien $\operatorname{rang} \sigma=1, \operatorname{Im} \sigma \simeq \mathcal{O}_{\mathbf{P}}(-n)$ et $\mathcal{N} / \operatorname{Im} \sigma$ sans torsion.

Démonstration. Quitte à changer $\mathcal{N}$ en $\mathcal{N}(n)$ on peut supposer $n=0$. Montrons $1 \Rightarrow 2$. L'hypothèse implique $q^{\sharp}(n) \geq 1$ et la proposition 1.3 montre alors que $\operatorname{rang} \sigma$ et $\operatorname{rang} \sigma(x)$ sont $\geq 1$. Supposons $\operatorname{rang} \sigma=1$. Comme $\operatorname{Im} \sigma / \operatorname{Im} s$ s'injecte dans $\mathcal{E}$, il est sans torsion. Comme $\operatorname{Im} \sigma$ et $\operatorname{Im} s \simeq \mathcal{O}_{\mathbf{P}}$ sont tous deux de rang 1 , ils sont égaux et on a la condition requise.

$2 \Rightarrow 1$. Si rang $\sigma=1$ on a $\operatorname{Im} \sigma \simeq \mathcal{O}_{\mathbf{P}}$ et on peut prendre pour $s$ n'importe quel élément non nul de $N_{0}^{\prime}$. 
Supposons $\operatorname{rang} \sigma \geq 2$. On considère le sous-schéma fermé réduit $Z$ de $\mathbf{P}\left(N_{0}^{\prime}\right) \times \mathbf{P}^{3}$ adhérence de l'ensemble des couples $(s, x)$ tels que $s(x)=0$. Si on note $d$ la dimension de $\mathbf{P}\left(N_{0}^{\prime}\right)$ il suffit de montrer qu'on a $\operatorname{dim} Z \leq d+1$. En effet, pour $s$ général dans $N_{0}^{\prime}$ la fibre $Z(s)$ sera de dimension $\leq 1$ ce qui assurera que Coker $s$ est localement libre en codimension 1, donc sans torsion, cf. [MDP1] IV 1.2.

Au point générique $\xi$ de $\mathbf{P}^{3}$ on a rang $\sigma(\xi) \geq 2$ donc la fibre $Z(\xi)$ est de dimension $\leq d-2$. En un point $x$ de codimension 1 on a rang $\sigma(x) \geq 1$ donc $\operatorname{dim} Z(x) \leq d-1$. Enfin, en un point de codimension $\geq 2$ les fibres sont de dimension $\leq d$. En définitive on a bien $\operatorname{dim} Z \leq d+1$.

Théorème 1.5. On reprend les notations de 1.0 et 1.1. Soit $\mathcal{P}$ un faisceau dissocié de fonction caractéristique $p$. Soit $H$ le sous-espace vectoriel de $\operatorname{Hom}_{\mathcal{O}_{\mathbf{P}}}(\mathcal{P}, \mathcal{N})$ formé des homomorphismes "à valeurs dans $N^{\prime}$ " (i.e., tels que l'on ait $\left.u\left(H_{*}^{0} \mathcal{P}\right) \subset N^{\prime}\right)$. Les conditions suivantes sont équivalentes:

1) Pour u général dans $H$, on a une suite exacte $0 \rightarrow \mathcal{P} \stackrel{u}{\longrightarrow} \mathcal{N} \rightarrow \mathcal{E} \rightarrow 0$ avec $\mathcal{E}$ sans torsion.

2) La fonction $p$ vérifie:

i) $p^{\sharp}(n) \leq q^{\sharp}(n)$ pour tout $n$,

ii) s'il existe $n \in \mathbf{Z}$ avec $p^{\sharp}(n)=q^{\sharp}(n)=\operatorname{rang} \sigma_{n}$, on a un isomorphisme $\mathcal{P}_{\leq n} \simeq \mathcal{N}_{N^{\prime}, \leq n}$.

Démonstration. 1) $\Rightarrow 2$ ). Comme $\mathcal{N} / \mathcal{P}_{\leq n}$ est sans torsion la condition i) résulte de la définition de la fonction $q$.

Montrons l'assertion ii). Soit $n$ vérifiant $p^{\sharp}(n)=q^{\sharp}(n)=\operatorname{rang} \sigma_{n}$. On a le diagramme commutatif de suites exactes

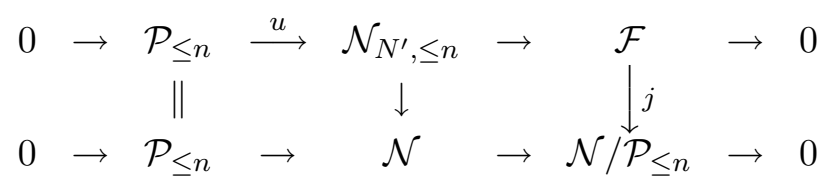

Comme $j$ est injective, le faisceau $\mathcal{F}=$ Coker $u$ est sans torsion. Mais, les faisceaux $\mathcal{P}_{\leq n}$ et $\mathcal{N}_{N^{\prime}, \leq n}$ sont tous deux de rang $p^{\sharp}(n)=q^{\sharp}(n)=\operatorname{rang} \sigma_{n}$, donc $\mathcal{F}$ est nul et $u$ est un isomorphisme.

$2) \Rightarrow 1$ ). On procède par récurrence sur le rang de $\mathcal{P}$. Le cas $\operatorname{rang} \mathcal{P}=0$ est trivial.

Supposons $\operatorname{rang} \mathcal{P} \geq 1$. Soit $a=\sup \mathcal{P}$. Si on a $p^{\sharp}(a)=q^{\sharp}(a)=\operatorname{rang} \sigma_{a}$, la condition ii) montre qu'on a un isomorphisme $\mathcal{P} \simeq \mathcal{N}_{N^{\prime}, \leq a}$. De plus, le quotient $\mathcal{N} / \mathcal{N}_{N^{\prime}, \leq a}$ est sans torsion. En effet, par définition de $q^{\sharp}(a)$, il existe un sous-faisceau dissocié $\mathcal{P}^{\prime}$ de $\mathcal{N}$, de $\operatorname{rang} q^{\sharp}(a)$, avec $\sup \mathcal{P}^{\prime} \leq a$ et $\mathcal{N} / \mathcal{P}^{\prime}$ sans torsion. On a donc une injection $j: \mathcal{P}^{\prime} \rightarrow \mathcal{N}_{N^{\prime}, \leq a}$. Comme ces faisceaux ont même rang, Coker $j$ est de torsion. Mais, par le lemme $\mathrm{du}$ serpent, Coker $j$ s'injecte dans $\mathcal{N} / \mathcal{P}^{\prime}$ qui est sans torsion, donc il est nul et $j$ est un isomorphisme. Si $u$ est un élément général de $H, u$ induit 
un isomorphisme de $\mathcal{P}$ sur $\mathcal{N}_{N^{\prime}, \leq a}$, donc Coker $u$ est sans torsion, d'où la conclusion.

Ce cas étant maintenant exclu, on considère la fonction $p^{\prime}$ définie par $p^{\prime}(n)=p(n)$ pour $n<a$ et $p^{\prime}(a)=p(a)-1$. Cette fonction vérifie les mêmes conditions que $p$ de sorte que si on pose $\mathcal{P}^{\prime}=\bigoplus_{n \in \mathbf{Z}} \mathcal{O}_{\mathbf{P}}(-n)^{p^{\prime}(n)}$ on peut appliquer l'hypothèse de récurrence à $\mathcal{P}^{\prime}$. Si $u^{\prime}$ est un homomorphisme général à valeurs dans $N^{\prime}$ on a donc une suite exacte:

$$
0 \rightarrow \mathcal{P}^{\prime} \stackrel{u^{\prime}}{\longrightarrow} \mathcal{N} \rightarrow \mathcal{E}^{\prime} \rightarrow 0
$$

avec $\mathcal{E}^{\prime}$ sans torsion. On note $E^{\prime}$ l'image de $N^{\prime}$ dans $H_{*}^{0} \mathcal{E}^{\prime}$ et on applique le lemme 1.4 à $\mathcal{E}^{\prime}$ et à l'entier $a$. En un point $x$ de codimension $\leq 1$ de $\mathbf{P}^{3}$ le faisceau $\mathcal{E}^{\prime}$ est localement libre et on a le diagramme commutatif de suites exactes:

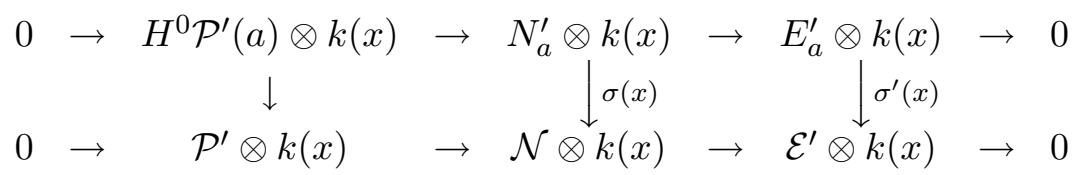

où l'on a posé $\sigma=\sigma_{\mathcal{N}, N^{\prime}, a}$ et $\sigma^{\prime}=\sigma_{\mathcal{E}^{\prime}, E^{\prime}, a}$.

En un tel point $x$ on a, en vertu de $1.3, \operatorname{rang} \sigma(x) \geq q^{\sharp}(a) \geq p^{\sharp}(a)$ et, comme $\mathcal{P}^{\prime}$ est de rang $p^{\sharp}(a)-1$, on en déduit rang $\sigma^{\prime}(x) \geq 1$. Si le rang de $\sigma^{\prime}$ était égal à 1 on aurait rang $\sigma=q^{\sharp}(a)=p^{\sharp}(a)$ or c'est le cas exclu ci-dessus.

On a donc rang $\sigma^{\prime} \geq 2$ et, en vertu de 1.4 on obtient, pour $v^{\prime}$ général dans $E_{a}^{\prime}$, une suite exacte

$$
0 \rightarrow \mathcal{O}_{\mathbf{P}}(-a) \stackrel{v^{\prime}}{\longrightarrow} \mathcal{E}^{\prime} \rightarrow \mathcal{E} \rightarrow 0
$$

avec $\mathcal{E}$ sans torsion; la flèche $v^{\prime}$ se relève en $v: \mathcal{O}_{\mathbf{P}}(-a) \rightarrow \mathcal{N}$ à valeurs dans $N^{\prime}$ et si on pose $u=v+u^{\prime}$, $u$ est général et on a Coker $u=\mathcal{E}$ comme annoncé.

Remarque 1.6. La condition 2) ii) du théorème signifie que le faisceau $\mathcal{N}_{N^{\prime}, \leq n}$ (isomorphe à $\mathcal{P}_{\leq n}$ ) est "obligatoire": c'est l'unique sous-faisceau $\mathcal{L}$ de $\mathcal{N}$, dissocié et de rang $q^{\sharp}(n)$, qui satisfait les conditions de 1.1: $\sup \mathcal{L} \leq n$ et $\mathcal{N} / \mathcal{L}$ sans torsion.

Proposition 1.7. On suppose $\mathcal{N}$ non dissocié et de rang $r$.

1) On a, pour tout $n, q^{\sharp}(n) \leq r-1$,

2) Si on suppose, de plus, que $N_{n}^{\prime}$ engendre $\mathcal{N}$ pour $n \gg 0$ on a $q^{\sharp}(n)=$ $r-1$ pour $n \gg 0$.

Démonstration. 1) Il est clair qu'on a $q^{\sharp}(n) \leq r-1$. En effet, si $\mathcal{P}$ est un sous-faisceau dissocié de rang $r$ de $\mathcal{N}$ (nécessairement distinct de $\mathcal{N}$ ), le quotient a de la torsion. 
2) On raisonne par récurrence sur $r$. Pour $r=1$ il n'y a rien à démontrer. Si $r \geq 2$ on prend $n \gg 0$ et on applique le lemme 1.4 à $\mathcal{N}$ et $n$. Par hypothèse on a rang $\sigma_{n}(x) \geq 2$ en tout point, d'où une section $s$ avec un quotient $\mathcal{E}$ sans torsion. Le module image $E^{\prime} \subset H_{*}^{0} \mathcal{E}$ satisfait les mêmes hypothèses que $N^{\prime}$. On a donc, par l'hypothèse de récurrence, $q^{\sharp} \mathcal{E}, E^{\prime}(n)=r-2$ pour $n$ grand, donc un sous-faisceau dissocié $\mathcal{P} \subset \mathcal{E}$, de rang $r-2$, à valeurs dans $E^{\prime}$ et à quotient sans torsion. La flèche $\mathcal{P} \rightarrow \mathcal{E}$ se relève à $\mathcal{N}$ et la considération du sous-faisceau $\mathcal{O}_{\mathbf{P}}(-n) \oplus \mathcal{P}$ de $\mathcal{N}$ montre qu'on a $q^{\sharp} \mathcal{N}, N^{\prime}(n) \geq r-1$.

c) Le cas des courbes.

Si on part d'un faisceau $\mathcal{N}$ réflexif et non dissocié on obtient le corollaire suivant:

Corollaire 1.8. On suppose que $\mathcal{N}$ est réflexif et non dissocié de rang $r$. Soit $\mathcal{P}$ un faisceau dissocié de rang $r-1$ et de fonction caractéristique $p$. Soit $H$ le sous-espace vectoriel de $\operatorname{Hom}_{\mathcal{O}_{\mathbf{P}}}(\mathcal{P}, \mathcal{N})$ formé des homomorphismes à valeurs dans $N^{\prime}$. Les conditions suivantes sont équivalentes:

i) Pour u général dans $H$, on a une suite exacte:

$$
0 \rightarrow \mathcal{P} \stackrel{u}{\longrightarrow} \mathcal{N} \rightarrow \mathcal{J}_{C}(h) \rightarrow 0
$$

où $C$ est une courbe de $\mathbf{P}^{3}$ et $h$ un entier.

ii) La fonction $p$ vérifie les deux conditions suivantes:

1) on a $p^{\sharp}(n) \leq q^{\sharp}(n)$ pour tout $n \in \mathbf{Z}$,

2) s'il existe $n$ tel que l'on ait $p^{\sharp}(n)=q^{\sharp}(n)=\operatorname{rang} \sigma_{n}$ on a un isomorphisme $\mathcal{P}_{\leq n} \simeq \mathcal{N}_{N^{\prime}, \leq n}$.

Il existe des fonctions $p$ vérifiant les conditions i) et ii) ci-dessus si et seulement si on a $q^{\sharp}(n)=r-1$ pour $n \gg 0$. Dans ce cas, la fonction $q$ elle-même convient et la valeur minimale du décalage $h$ correspond au cas $p=q$ et est égale à $\sum_{n \in \mathbf{Z}} n q(n)+\operatorname{deg} \mathcal{N}$.

Démonstration. Le sens i) $\Rightarrow$ ii) vient de 1.5. Réciproquement, on a, en vertu de 1.5 , un homomorphisme injectif $u: \mathcal{P} \rightarrow \mathcal{N}$ dont le conoyau $\mathcal{E}$ est un faisceau sans torsion de rang 1 donc l'idéal tordu $\mathcal{J}_{Z}(h)$ d'un sous-schéma $Z$ de $\mathbf{P}^{3}$ de dimension $\leq 1$. Comme $\mathcal{N}$ n'est pas dissocié, $Z$ n'est pas vide. Par ailleurs, comme $\mathcal{N}$ (resp. $\mathcal{P}$ ) est réflexif on a prof $\mathcal{J}_{Z, x} \geq 2$ en tout point fermé de $\mathbf{P}^{3}$, donc prof $\mathcal{O}_{Z, x} \geq 1$ et, a fortiori $\operatorname{dim} \mathcal{O}_{Z, x} \geq 1$, de sorte que $Z$ est une courbe.

S'il existe une fonction $p$ vérifiant les conditions ci-dessus, on a $p^{\sharp}(n)=$ $r-1$ pour $n \gg 0$ donc aussi $q^{\sharp}(n)=r-1$ pour $n \gg 0$ par ii) et 1.7. Réciproquement, si on a cette condition, il existe, par définition de $q$, un sous-faisceau $\mathcal{P}$ de $\mathcal{N}$, dissocié de fonction caractéristique $q$ dont le quotient $\mathcal{N} / \mathcal{P}$ est sans torsion de rang 1 , donc, par le même argument que ci-dessus, c'est un idéal tordu, de sorte que $q$ convient. Enfin, la condition $p^{\sharp} \leq q^{\sharp}$, 
jointe à la formule $\sum_{n \in \mathbf{Z}} n q(n)=-\sum_{n \in \mathbf{Z}} q^{\sharp}(n)$, montre que le décalage est minimal dans le cas de la fonction $q$.

\section{Remarques 1.9.}

1) L'existence d'un faisceau dissocié $\mathcal{P}$ vérifiant les conditions de 1.8 dépend essentiellement du sous-module $N^{\prime}$. Précisément, on a vu ci-dessus que cette existence équivaut à la condition $q^{\sharp}{ }_{\mathcal{N}, N^{\prime}}(n)=r-1$ pour $n \gg 0$. D'après 1.7 cette condition est satisfaite si $N_{n}^{\prime}$ engendre $\mathcal{N}$ pour $n \gg 0$. Dans certains cas il n'y a pas de faisceau $\mathcal{P}$ vérifiant les conditions de 1.8 (par exemple si $N^{\prime}$ est nul et $\mathcal{N}$ de rang $\geq 2$ ).

2) Si le faisceau $\mathcal{N}$ est dissocié le corollaire est en défaut lorsque $u$ (à valeurs dans $N^{\prime}$ ) identifie $\mathcal{P}$ à un facteur direct de $\mathcal{N}$ car le quotient est alors un faisceau $\mathcal{O}_{\mathbf{P}}(n)$. Dans le cas $N^{\prime}=N$ la fonction $q$ est la fonction caractéristique de $\mathcal{N}$ et on vérifie facilement que le seul cas à écarter est celui où l'on a $p(n) \leq q(n)$ pour tout $n$.

d) Calcul de la fonction $q$.

Définition 1.10. Avec les notations de 1.0 on pose $\alpha_{n}=\alpha_{n}\left(\mathcal{N}, N^{\prime}\right)=$ rang $\sigma_{n}$ et

$$
\beta_{n}=\beta_{n}\left(\mathcal{N}, N^{\prime}\right)=\operatorname{Min}\left\{\operatorname{rang} \sigma_{n}(x) \mid x \in \mathbf{P}^{3}, x \text { de codimension } 1\right\} .
$$

Remarque 1.11. On a $\beta_{n} \leq \alpha_{n} \leq \operatorname{rang} \mathcal{N}$. En vertu de 1.3 on a aussi $q^{\sharp}(n) \leq \beta_{n} \leq \alpha_{n}$.

La proposition suivante étudie le cas d'égalité $q^{\sharp}(n)=\alpha_{n}$ et le phénomène déjà rencontré en 1.5 (l'existence d'une partie "obligatoire" pour $\mathcal{N}$ ):

Proposition 1.12. Soit $a \in \mathbf{Z}$. On suppose qu'on a $q^{\sharp}(a)=\alpha_{a}$, c'est-à-dire qu'il existe un sous-faisceau dissocié $\mathcal{L} \subset \mathcal{N}$ vérifiant $H_{*}^{0} \mathcal{L} \subset N^{\prime}, \sup \mathcal{L} \leq a$, $\operatorname{rang} \mathcal{L}=\alpha_{a}=\operatorname{rang} \sigma_{a}$ et $\mathcal{N} / \mathcal{L}$ sans torsion. Alors, on a nécessairement $\mathcal{L}=\mathcal{N}_{N^{\prime}, \leq a}$ et, en posant $L=H_{*}^{0} \mathcal{L}, L=N_{\leq a}^{\prime}$. Si l est la fonction caractéristique de $\mathcal{L}$ on a $l^{\sharp}(n)=\alpha_{n}=\beta_{n}=q^{\sharp}(n)$ pour $n \leq a$ et $l^{\sharp}(n)=\alpha_{a}$ pour $n>a$.

Démonstration. On pose $\mathcal{K}=\mathcal{N} / \mathcal{L}$ et le lemme du serpent montre que dans le diagramme:

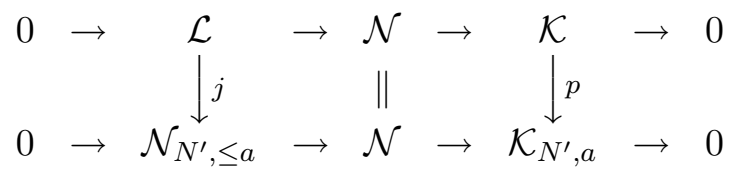

le noyau $\mathcal{F}$ de $p$ est isomorphe au conoyau de $j$. Comme $\mathcal{K}$ est sans torsion il en est de même de $\mathcal{F}$, ce qui, puisque $\mathcal{L}$ et $\mathcal{N}_{N^{\prime}, \leq a}$ sont tous deux de rang $\alpha_{a}$, montre que $j$ est un isomorphisme. Comme $L=H_{*}^{0} \mathcal{L}$ est inclus dans $N^{\prime}$, donc aussi dans $N_{\leq a}^{\prime}$, cela montre aussi l'égalité $L=N_{\leq a}^{\prime}$. 
Comme $L$ est libre, on en déduit, pour $n \leq a, L_{\leq n}=N_{\leq n}^{\prime}$, d'où $l^{\sharp}(n)=$ $\operatorname{rang} L_{\leq n}=\operatorname{rang} N_{\leq n}^{\prime}=\alpha_{n}=\beta_{n}$. Il est clair que $\mathcal{N} / \mathcal{L}_{\leq n}$ est sans torsion et on a donc aussi $\alpha_{n}=q^{\sharp}(n)$. Enfin, comme sup $L \leq a$, on a $l^{\sharp}(n)=l^{\sharp}(a)=\alpha_{a}$ pour $n>a$.

Comme $\mathcal{N}$ est sans torsion tout entier $n \ll 0$ vérifie la condition $q^{\sharp}(n)=$ $\alpha_{n}=0$ et on peut donc poser la définition suivante:

Définition 1.13. Avec les notations de 1.0, on définit l'entier $b_{0}=$ $b_{0}\left(\mathcal{N}, N^{\prime}\right)^{1}$ comme le plus grand entier $n$ qui vérifie $q^{\sharp}(n)=\operatorname{rang} \sigma_{n}=\alpha_{n}$. Si tous les entiers $n$ conviennent on pose $b_{0}=+\infty$.

La proposition suivante, qui est immédiate à partir de 1.12, sera utile pour le calcul explicite de $b_{0}$ :

Proposition 1.14. Soit $a \in \mathbf{Z}$. Les conditions suivantes sont équivalentes:

i) on $a \quad a \leq b_{0}$,

ii) on $a q^{\sharp}(a)=\alpha_{a}$,

iii) il existe un sous-faisceau dissocié $\mathcal{L} \subset \mathcal{N}$ vérifiant $H_{*}^{0} \mathcal{L} \subset N^{\prime}, \sup \mathcal{L} \leq$ $a$, rang $\mathcal{L}=\operatorname{rang} \sigma_{a}$ et $\mathcal{N} / \mathcal{L}$ sans torsion,

iv) le sous-module $N_{\leq a}^{\prime}$ de $N^{\prime}$ est libre de rang $\alpha_{a}$ et le quotient $N / N_{\leq a}^{\prime}$ est sans torsion.

Théorème 1.15. La fonction $q$ se calcule comme suit:

1) pour $n \leq b_{0}$ on $a q^{\sharp}(n)=\alpha_{n}=\beta_{n}$,

2) pour $n>b_{0}$ on a $q^{\sharp}(n)=\inf \left(\alpha_{n}-1, \beta_{n}\right)$.

Démonstration. L'assertion 1) résulte de 1.12. Supposons $n>b_{0}$ (donc $\mathcal{N} \neq 0)$. On a $q^{\sharp}(n)<\alpha_{n}$ par définition de $b_{0}$ et $q^{\sharp}(n) \leq \beta_{n}$ par 1.11, donc $q^{\sharp}(n) \leq \inf \left(\alpha_{n}-1, \beta_{n}\right)$. Réciproquement, posons $d_{n}=d_{n}\left(\mathcal{N}, N^{\prime}\right)=$ $\inf \left(\alpha_{n}-1, \beta_{n}\right)$ et montrons, par récurrence sur le rang de $\mathcal{N}$, qu'on a $q^{\sharp}(n) \geq d_{n}$, c'est-à-dire qu'on a un sous-faisceau dissocié $\mathcal{P}$ de $\mathcal{N}$, en degrés $\leq n$, de rang $d_{n}$, à quotient sans torsion. Si $d_{n}$ est nul il n'y a rien à montrer. Supposons $d_{n} \geq 1$. En vertu de 1.4 il existe une suite exacte $0 \rightarrow \mathcal{O}_{\mathbf{P}}(-n) \stackrel{s}{\longrightarrow} \mathcal{N} \rightarrow \mathcal{E} \rightarrow 0$ avec $s$ à valeurs dans $N^{\prime}$ et $\mathcal{E}$ sans torsion. Soit $E^{\prime}$ l'image de $N^{\prime}$ dans $H_{*}^{0} \mathcal{E}$. On a le lemme suivant:

Lemme 1.16. On a $d_{n}\left(\mathcal{E}, E^{\prime}\right) \geq d_{n}\left(\mathcal{N}, N^{\prime}\right)-1$.

Démonstration. (de 1.16) Soit $x$ un point de codimension $\leq 1$ de $\mathbf{P}^{3}$. On a le diagramme de suites exactes suivant:

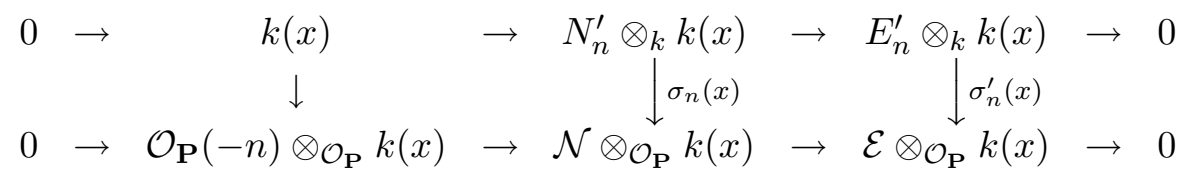

\footnotetext{
${ }^{1}$ Par rapport aux notations usuelles de [MDP1] 1,3 on a posé $b_{0}=a_{0}-1$.
} 
dont on déduit l'inégalité $\operatorname{rang} \sigma_{n}^{\prime}(x) \geq \operatorname{rang} \sigma_{n}(x)-1$, d'où $\alpha_{n}\left(\mathcal{E}, E^{\prime}\right) \geq$ $\alpha_{n}\left(\mathcal{N}, N^{\prime}\right)-1$ et $\beta_{n}\left(\mathcal{E}, E^{\prime}\right) \geq \beta_{n}\left(\mathcal{N}, N^{\prime}\right)-1$ et la conclusion du lemme.

On peut alors finir de prouver 1.15. En vertu de l'hypothèse de récurrence on a, dans tous les cas, $q^{\sharp} \mathcal{E}, E^{\prime}(n) \geq d_{n}\left(\mathcal{E}, E^{\prime}\right) \geq d_{n}\left(\mathcal{N}, N^{\prime}\right)-1$. Par définition de la fonction $q$ cela signifie qu'il existe un sous-faisceau dissocié $\mathcal{P}^{\prime}$ de $\mathcal{E}$, de rang $\geq d_{n}\left(\mathcal{N}, N^{\prime}\right)-1$, en degrés $\leq n$, avec $\mathcal{E} / \mathcal{P}^{\prime}$ sans torsion. La flèche $\mathcal{P}^{\prime} \rightarrow \mathcal{E}$ se relève à $\mathcal{N}$ et $\mathcal{O}_{\mathbf{P}}(-n) \oplus \mathcal{P}^{\prime}$ fournit le faisceau $\mathcal{P}$ cherché.

Remarque 1.17. En vertu de 1.12 on peut remplacer la condition ii) 2) de 1.8 par la suivante:

S'il existe un entier $n_{0}$ (nécessairement $\leq b_{0}$ ) tel que $p^{\sharp}\left(n_{0}\right)=q^{\sharp}\left(n_{0}\right)=$ rang $\sigma_{n_{0}}$ on a $p(n)=q(n)$ pour tout $n \leq n_{0}$.

\section{Construction de familles de courbes.}

Dans tout ce paragraphe on travaille sur un anneau noethérien local $A$ d'idéal maximal $m_{A}$. On pose $T=\operatorname{Spec} A$ et on désigne par $t$ le point fermé de $T$ et par $k(t)$ le corps résiduel. On suppose $k(t)$ infini, cf. 2.11 pour une variante lorsque $k(t)$ est fini.

a) Résultats préliminaires.

Définition 2.1. On dit que le faisceau $\mathcal{N}$ est sans torsion dans la fibre spéciale si le faisceau $\mathcal{N}_{t}$ est sans torsion.

Proposition 2.2. On suppose qu'on a une suite exacte $\mathcal{F} \stackrel{u}{\longrightarrow} \mathcal{G} \rightarrow \mathcal{H} \rightarrow 0$ de faisceaux cohérents sur $\mathbf{P}_{A}^{3}$ avec $\mathcal{G}$ plat sur $A^{2}$ et $\mathcal{F}$ dissocié. Alors, les conditions suivantes sont équivalentes:

a) $u$ est injectif et $\mathcal{H}$ est plat sur $A$,

b) $u_{t}: \mathcal{F}_{t} \rightarrow \mathcal{G}_{t}$ est injectif au point fermé $t$ de $T$.

Démonstration. Cela résulte du critère local de platitude, cf. [EGA] $0_{I I I}$ 10.2.4 et de [EGA] IV, 11, Note de la page 118 .

Nous faisons maintenant le lien avec la situation du paragraphe 1.

Soit $\mathcal{N}$ un faisceau cohérent sur $\mathbf{P}_{A}^{3}$ et soit $\rho: H_{*}^{0}\left(\mathbf{P}_{A}^{3}, \mathcal{N}\right) \otimes_{A} k(t) \rightarrow$ $H_{*}^{0}\left(\mathbf{P}_{k(t)}^{3}, \mathcal{N}_{t}\right)$ l'homomorphisme canonique. On note $N^{\prime}$ l'image de $\rho$ dans $N=H_{*}^{0} \mathcal{N}_{t}$. En général $N^{\prime}$ est différent de $N$ (dire que $N^{\prime}=N$ signifie que $H_{*}^{0} \mathcal{N}$ commute au changement de base, cf. [AG] III 12.11). Dans toute la suite nous utilisons les résultats du paragraphe 1 appliqués au faisceau $\mathcal{N}_{t}$ et au sous-module $N^{\prime}$. On notera que, si $\mathcal{N}$ est plat sur $A$ et $n \gg 0, H^{0} \mathcal{N}(n)$ commute au changement de base, donc que $N_{n}^{\prime}$ engendre $\mathcal{N}_{t}$, cf. 1.7.

\footnotetext{
${ }^{2}$ Dans ce paragraphe, la platitude est toujours sous-entendue sur $A$.
} 
Corollaire 2.3. Soit $\mathcal{N}$ un faisceau cohérent sur $\mathbf{P}_{A}^{3}$, plat et sans torsion dans la fibre spéciale, soit $N^{\prime}=\operatorname{Im} \rho$ comme ci-dessus et soit $\mathcal{P}$ un faisceau dissocié sur $\mathbf{P}_{A}^{3}$ de fonction caractéristique $p$. Les propriétés suivantes sont équivalentes:

1) Il existe une suite exacte de faisceaux $\operatorname{sur} \mathbf{P}_{A}^{3}$

$$
0 \rightarrow \mathcal{P} \stackrel{u}{\longrightarrow} \mathcal{N} \rightarrow \mathcal{E} \rightarrow 0
$$

avec $\mathcal{E}$ plat et sans torsion dans la fibre spéciale.

2) Il existe une suite exacte de faisceaux sur $\mathbf{P}_{k(t)}^{3}$

$$
0 \rightarrow \mathcal{P}_{t} \stackrel{u_{0}}{\longrightarrow} \mathcal{N}_{t} \rightarrow \mathcal{E}_{0} \rightarrow 0
$$

avec $u_{0}$ à valeurs dans $N^{\prime}$ et $\mathcal{E}_{0}$ sans torsion.

Démonstration. Le sens 1) $\Rightarrow 2$ ) est immédiat en tensorisant par $k(t)$ puisque $\mathcal{E}$ est plat sur $A$ (comme $u_{t}$ provient de $u$ il est bien à valeurs dans $\left.N^{\prime}\right)$. Dans l'autre sens, comme $P_{t}=H_{*}^{0} \mathcal{P}_{t}$ est libre sur $R$, la flèche $P_{t} \rightarrow N^{\prime}$ se relève en $P_{t} \rightarrow H_{*}^{0} \mathcal{N} \otimes_{A} k(t)$ puis en $P \rightarrow H_{*}^{0} \mathcal{N}$ et induit $u: \mathcal{P} \rightarrow \mathcal{N}$ avec $u_{t}=u_{0}$. On conclut avec 2.2 .

b) Construction de familles de courbes.

Nous définissons les invariants associés à un faisceau $\mathcal{N}$ sur $\mathbf{P}_{A}^{3}$; ce sont ceux du faisceau $\mathcal{N}_{t}$ relatifs au sous-module $N^{\prime}$ :

Définition 2.4. Soit $\mathcal{N}$ un faisceau cohérent sur $\mathbf{P}_{A}^{3}$, plat et sans torsion dans la fibre spéciale. On considère l'homomorphisme canonique $\rho$ : $H_{*}^{0}\left(\mathbf{P}_{A}^{3}, \mathcal{N}\right) \otimes_{A} k(t) \rightarrow H_{*}^{0}\left(\mathbf{P}_{k(t)}^{3}, \mathcal{N}_{t}\right)$ et on note $N^{\prime}$ son image.

On pose alors $\mathcal{N}_{t, \leq n}=\left(\mathcal{N}_{t}\right)_{N^{\prime}, \leq n}, \alpha_{n}(\mathcal{N})=\alpha_{n}\left(\mathcal{N}_{t}, N^{\prime}\right), \beta_{n}(\mathcal{N})=$ $\beta_{n}\left(\mathcal{N}_{t}, N^{\prime}\right), b_{0}(\mathcal{N})=b_{0}\left(\mathcal{N}_{t}, N^{\prime}\right), q_{\mathcal{N}}=q_{\mathcal{N}_{t}, N^{\prime}}$. S'il n'y a pas d'ambiguïté sur le faisceau $\mathcal{N}$ ces invariants seront notés simplement $\alpha_{n}, \beta_{n}, b_{0}, q$.

Soient $H$ un $A$-module de type fini, $\bar{H}=H \otimes_{A} k(t)$ le $k(t)$-espace vectoriel de dimension finie obtenu par réduction modulo $m_{A}$ et $\pi: H \rightarrow \bar{H}$ la projection canonique. Nous dirons qu'une propriété $P$ des éléments de $H$ est vraie pour $h$ "général" dans $H$ s'il existe un ouvert de Zariski non vide $\bar{U}$ du schéma affine associé à $\bar{H}$ tel que $P$ soit vraie pour tout $h \in \pi^{-1}(\bar{U})$. Puisque $k(t)$ est infini, un tel ouvert a des points rationnels, et son image réciproque $\pi^{-1}(\bar{U})$, considérée comme sous-ensemble du $A$-module $H$, n'est pas vide.

Avec ces notations et compte tenu de la définition de $b_{0}$, le corollaire 2.3, joint à 1.5, donne aussitôt le théorème suivant:

Théorème 2.5. On suppose que le faisceau $\mathcal{N}$ est plat et sans torsion dans la fibre spéciale. Soit $\mathcal{P}$ un faisceau dissocié de fonction caractéristique $p$. Les conditions suivantes sont équivalentes: 
i) Pour u général dans $H=\operatorname{Hom}_{\mathcal{O}_{\mathbf{P}}}(\mathcal{P}, \mathcal{N})$, on a une suite exacte

$$
0 \rightarrow \mathcal{P} \stackrel{u}{\longrightarrow} \mathcal{N} \rightarrow \mathcal{E} \rightarrow 0
$$

où $\mathcal{E}$ est plat et sans torsion dans la fibre spéciale.

ii) La fonction $p$ vérifie les conditions 1) et 2) ci-dessous:

1) on $a p^{\sharp}(n) \leq q^{\sharp}(n)$ pour tout $n \in \mathbf{Z}$,

2) s'il existe $n \leq b_{0}$ tel que l'on ait $p^{\sharp}(n)=q^{\sharp}(n)$ on a un isomorphisme $\left(\mathcal{P}_{\leq n}\right)_{t} \simeq \mathcal{N}_{t, \leq n}$.

La fonction $q$ vérifie les conditions i) et ii) ci-dessus.

Dans le cas des courbes on a le résultat suivant:

Théorème 2.6. Soit $\mathcal{N}$ un faisceau sur $\mathbf{P}_{A}^{3}$, localement libre de rang $r$ et non dissocié. Soit $\mathcal{P}=\bigoplus_{n \in \mathbf{Z}} \mathcal{O}_{\mathbf{P}_{A}}(-n)^{p(n)}$ un faisceau dissocié de rang $r-1$. Alors, les conditions suivantes sont équivalentes:

i) Pour u général dans $H=\operatorname{Hom}_{\mathcal{O}_{\mathbf{P}}}(\mathcal{P}, \mathcal{N})$, on a une suite exacte:

$$
0 \rightarrow \mathcal{P} \stackrel{u}{\longrightarrow} \mathcal{N} \rightarrow \mathcal{J}_{\mathcal{C}}(h) \rightarrow 0
$$

où $\mathcal{C}$ est une famille de courbes de $\mathbf{P}_{A}^{3}$, plate sur $A$.

ii) La fonction $p$ vérifie les conditions suivantes:

1) on a $p^{\sharp}(n) \leq q^{\sharp}(n)$ pour tout $n \in \mathbf{Z}$,

2) s'il existe $n \leq b_{0}$ tel que l'on ait $p^{\sharp}(n)=q^{\sharp}(n)$ on a un isomorphisme $\left(\mathcal{P}_{\leq n}\right)_{t} \simeq \mathcal{N}_{t, \leq n}$.

La fonction $q$ vérifie les conditions i) et ii) ci-dessus. La valeur minimale $d u$ décalage $h$ est égale à $\sum_{n \in \mathbf{Z}} n q(n)+\operatorname{deg} \mathcal{N}$. La famille de courbes correspondant à ce décalage minimum est donnée par une résolution comme ci-dessus, avec $p=q$.

Démonstration. Il reste à prouver ii) $\Rightarrow$ i). En vertu de 2.5 on a un homomorphisme injectif $u: \mathcal{P} \rightarrow \mathcal{N}$ dont le conoyau $\mathcal{E}$ est un faisceau de rang 1 , plat et sans torsion dans la fibre spéciale. Par ailleurs, comme $\mathcal{N}$ (resp. $\mathcal{P}$ ) est localement libre de rang $r$ (resp. $r-1$ ) on a un isomorphisme $\mathcal{N}(-d) \simeq \wedge^{r-1} \mathcal{N}^{\vee}\left(\right.$ resp. $\left.\mathcal{O}_{\mathbf{P}_{A}^{3}}(-e) \simeq \wedge^{r-1} \mathcal{P}^{\vee}\right)$ où $d$ (resp. e) est le degré de $\mathcal{N}$ (resp. $\mathcal{P}$ ). On en déduit un complexe

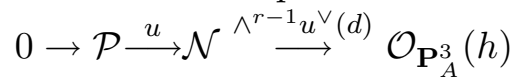

avec $h=d-e$. Le conoyau de $\wedge^{r-1} u^{\vee}(d)$ est de la forme $\mathcal{O}_{\mathcal{C}}(h)$ où $\mathcal{C}$ est un sous-schéma fermé de $\mathbf{P}_{A}^{3}$. Comme $\mathcal{E}$ est sans torsion dans la fibre spéciale il résulte du théorème de Hilbert-Burch, cf. par exemple [MDP1] II, 1.1, que le complexe ci-dessus est une suite exacte dans la fibre spéciale, donc une suite exacte sur $\mathbf{P}_{A}^{3}$ en vertu de 2.2 appliqué à la suite $\mathcal{E} \stackrel{u}{\longrightarrow} \mathcal{O}_{\mathbf{P}_{A}^{3}}(h) \rightarrow$ $\mathcal{O}_{\mathcal{C}}(h) \rightarrow 0$. De plus, comme $\mathcal{N}$ n'est pas dissocié, $\mathcal{C}$ est non vide donc est une famille de courbes (cf. [MDP3] III 1.7) plate sur $A$ en vertu du critère local de platitude. 
Comme $N_{n}^{\prime}$ engendre $\mathcal{N}_{t}$ pour $n \gg 0$ on a $q^{\sharp}(n)=r-1$ pour $n \gg 0$ (cf. 1.9.1), de sorte que $q$ vérifie les conditions de 2.6. Enfin, la condition $p^{\sharp} \leq q^{\sharp}$, jointe à la formule $\sum_{n \in \mathbf{Z}} n q(n)=-\sum_{n \in \mathbf{Z}} q^{\sharp}(n)$, montre que le décalage est minimal dans le cas de la fonction $q$.

Corollaire 2.7. Soit $\mathcal{N}$ un faisceau localement libre de $\operatorname{rang} r \operatorname{sur} \mathbf{P}_{A}^{3}$. Il existe une famille $\mathcal{C}$ de courbes lisses et connexes de $\mathbf{P}_{A}^{3}$, plate sur $A$, et un entier $h$ tels que $\mathcal{C}$ admette une résolution de type $N$ de la forme:

$$
0 \rightarrow \mathcal{P} \rightarrow \mathcal{N} \rightarrow \mathcal{J}_{\mathcal{C}}(h) \rightarrow 0 .
$$

Démonstration. On choisit un entier $n$ assez grand pour que $H^{0} \mathcal{N}(n)$ commute au changement de base et que $\mathcal{N}(n-1)$ soit engendré par ses sections globales. On pose $\mathcal{P}=\mathcal{O}_{\mathbf{P}}(-n-1)^{r-1}$. On montre comme cidessus qu'un homomorphisme général $u: \mathcal{P} \rightarrow \mathcal{N}$ a pour conoyau l'idéal tordu d'une famille plate de courbes $\mathcal{C}$ (comme les degrés dans $\mathcal{P}$ ont été choisis assez grands il n'est pas nécessaire de supposer $\mathcal{N}$ non dissocié ici). De plus, les hypothèses montrent que la courbe spéciale est lisse connexe (en toute caractéristique), cf. [K] Th. 3.3, donc aussi les autres.

c) Application à la biliaison.

Nous décrivons maintenant les familles de courbes qui sont minimales dans les classes de biliaison.

Rappelons d'abord, cf. [HMDP1] que les classes de biliaison correspondent, via les résolutions de type $N$, aux classes de faisceaux localement libres pour la relation de pseudo-isomorphisme et qu'une telle classe contient un faisceau localement libre $\mathcal{N}_{0}$ extraverti (c'est-à-dire vérifiant $\left.\operatorname{Ext}_{R_{A}}^{1}\left(H_{*}^{0} \mathcal{N}_{0}, R_{A}\right)=H_{*}^{1} \mathcal{N}_{0}^{\vee}=0\right)$, minimal (i.e. sans facteur direct dissocié), unique à isomorphisme près, cf. [HMDP1] 2.14.

Nous aurons besoin aussi de la notion de déformation à cohomologie uniforme:

Définition 2.8. Posons $S=\operatorname{Spec} A[\lambda]$ et soit $\Omega$ un ouvert de $S$. On note $A_{\Omega}$ l'ensemble des $a \in A$ tels que la section $\lambda=a$ soit contenue dans $\Omega$. Soit $\mathcal{C} \rightarrow \Omega$ une famille plate de courbes. Pour $a \in A_{\Omega}$ on note $\mathcal{C}_{a}$ la famille de courbes (paramétrée par $A$ ) correspondante. Si les points $a$ et $b$ sont dans $A_{\Omega}$ on dit que $\mathcal{C}$ est une déformation joignant $\mathcal{C}_{a}$ et $\mathcal{C}_{b}$. On dit que cette déformation est à cohomologie uniforme si, pour tout $s \in \operatorname{Spec} A$, tout $i=0,1,2$ et tout $n \in \mathbf{Z}$ la fonction $a \rightarrow h^{i}\left(\mathcal{J}_{\mathcal{C}_{a}} \otimes_{A} k(s)\right)(n)$ est constante sur $A_{\Omega}$ (mais attention, cette dimension peut varier avec $s$ ).

Avec ces notations on a le résultat suivant:

Corollaire 2.9. Soit $\mathcal{N}_{0}$ un faisceau localement libre extraverti minimal. Posons $q=q_{\mathcal{N}_{0}}$ et $h_{0}=\sum_{n \in \mathbf{Z}} n q(n)+\operatorname{deg} \mathcal{N}_{0}$.

1) Il existe une famille de courbes $\mathcal{C}_{0}$ et une résolution $0 \rightarrow \mathcal{P}_{0} \stackrel{v}{\longrightarrow} \mathcal{N}_{0} \rightarrow$ $\mathcal{J}_{\mathcal{C}_{0}}\left(h_{0}\right) \rightarrow 0$ avec $\mathcal{P}_{0}$ dissocié. 
2) Si $\mathcal{C}_{1}$ est une famille de courbes de la classe de biliaison de $\mathcal{C}_{0}$, elle admet une résolution $0 \rightarrow \mathcal{P} \rightarrow \mathcal{N}_{0} \oplus \mathcal{L} \rightarrow \mathcal{J}_{\mathcal{C}_{1}}(h) \rightarrow 0$ avec $\mathcal{L}$ dissocié et on on a $h \geq h_{0}$. Si d et $g$ (resp. $d_{0}$ et $g_{0}$ ) sont respectivement le degré et le genre de $\mathcal{C}_{1}$ (resp. $\left.\mathcal{C}_{0}\right)$ on a $d \geq d_{0}$ et $g \geq g_{0}$.

3) Réciproquement, pour tout $h \geq h_{0}$, il existe une famille de courbes $\mathcal{C}_{1}$ avec une résolution comme ci-dessus.

4) Si on a $h=h_{0}$, les familles $\mathcal{C}_{0}$ et $\mathcal{C}_{1}$ sont jointes par une déformation à cohomologie uniforme. On dit que $\mathcal{C}_{0}$ est une famille minimale de courbes de la classe de biliaison associée à $\mathcal{N}_{0}$.

Démonstration. L'existence de $\mathcal{C}_{0}$ et de la suite exacte vient de 2.6. Le faisceau $\mathcal{P}_{0}$ est un faisceau dissocié de fonction caractéristique $q$.

Soit $\mathcal{C}_{1}$ une famille de courbes de la classe de biliaison de $\mathcal{C}_{0}$. D'après [HMDP1] 2.22 il existe une résolution de type $N$ extravertie de $\mathcal{C}_{1}$, avec un faisceau $\mathcal{N}$. D'après [HMDP1] 3.2, ce faisceau est pseudo-isomorphe à $\mathcal{N}_{0}\left(h^{\prime}\right)$ pour un décalage $h^{\prime}$ convenable. Il résulte alors de [HMDP1] 2.14 que l'on a (à décalage près) $\mathcal{N}=\mathcal{N}_{0} \oplus \mathcal{L}$ avec $\mathcal{L}$ dissocié de fonction caractéristique $l$. On vérifie aussitôt la formule $q_{\mathcal{N}^{\sharp}}(n)=q_{\mathcal{N}_{0}}{ }^{\sharp}(n)+l^{\sharp}(n)$ (cf. [MDP1] IV 2.9) et on en déduit $h \geq h_{0}$. Les assertions sur degré et genre résultent de [MDP1] IV 5.2 et 5.3 appliqués au point $t$.

Le point 3) s'obtient en faisant des biliaisons élémentaires triviales, cf. [HMDP1] 1.6, à partir de $\mathcal{C}_{0}$.

Supposons maintenant $h=h_{0}$. On a une suite exacte $0 \rightarrow \mathcal{P} \stackrel{u}{\longrightarrow} \mathcal{N}_{0} \oplus \mathcal{L} \rightarrow$ $\mathcal{J}_{\mathcal{C}_{1}}\left(h_{0}\right) \rightarrow 0$ et, comme $h_{0}$ est le minimum possible, le faisceau dissocié $\mathcal{P}$ a pour fonction caractéristique $q_{\mathcal{N}}$ (cf. 2.6), donc s'écrit $\mathcal{P}=\mathcal{P}_{0} \oplus \mathcal{L}$ avec $\mathcal{P}_{0}$ comme ci-dessus.

Posons $u={ }^{t}\left(u_{1}, u_{2}\right)$ où $u_{2}$ va de $\mathcal{P}_{0} \oplus \mathcal{L}$ dans $\mathcal{L}$. Nous allons montrer que $u_{2}$ admet une section. Par récurrence sur le rang de $\mathcal{L}$ on se ramène au cas $\mathcal{L}=\mathcal{O}_{\mathbf{P}_{A}^{3}}(-a)$ et il faut montrer que la matrice $u_{2}$ a un coefficient qui est une constante inversible. On a la suite exacte

$$
0 \rightarrow\left(\mathcal{P}_{0}\right)_{\leq a} \oplus \mathcal{O}_{\mathbf{P}_{A}^{3}}(-a) \stackrel{w}{\longrightarrow} \mathcal{N}_{0} \oplus \mathcal{O}_{\mathbf{P}_{A}^{3}}(-a) \rightarrow \mathcal{E} \rightarrow 0
$$

où $\mathcal{E}$, qui est extension de $\mathcal{J}_{\mathcal{C}_{1}}\left(h_{0}\right)$ par le faisceau dissocié $\mathcal{P}_{>a}$, est encore plat et sans torsion dans la fibre spéciale. Posons $w={ }^{t}\left(w_{1}, w_{2}\right)$ et soit $\mathcal{F}=$ Coker $w_{1}$. Si $w_{2}$ n'a pas de coefficient constant inversible elle est nulle au point fermé $t$ et on en déduit que $\left(w_{1}\right)_{t}$ est injective et que $\mathcal{F}_{t}$ est facteur direct de $\mathcal{E}_{t}$, donc sans torsion. Mais, cela implique $q_{\mathcal{N}_{0}}{ }^{\sharp}(a)+1 \leq q_{\mathcal{N}_{0}}{ }^{\sharp}(a)$, ce qui est absurde.

L'existence de la section de $u_{2}$ permet de "simplifier" les facteurs $\mathcal{L}$ c'està-dire d'obtenir une suite exacte $0 \rightarrow \mathcal{P}_{0} \stackrel{u}{\longrightarrow} \mathcal{N}_{0} \rightarrow \mathcal{J}_{\mathcal{C}_{1}}\left(h_{0}\right) \rightarrow 0$.

On considère alors l'homomorphisme $w_{\lambda}=\lambda u+(1-\lambda) v: \mathcal{P}_{0} \otimes_{A} A[\lambda] \rightarrow$ $\mathcal{N}_{0} \otimes_{A} A[\lambda]$ et son image $\bar{w}_{\lambda}=\lambda \bar{u}+(1-\lambda) \bar{v}$ au point fermé. En vertu de 2.6 et de la définition de "général", il existe un ouvert affine $\Omega=\operatorname{Spec} \Lambda$ 
de la droite affine Spec $A[\lambda]$, contenant les points 0 et 1 de la fibre fermée, donc aussi les sections $\lambda=0$ et $\lambda=1$, tel que, sur cet ouvert, $w_{\lambda}$ définit une famille plate de courbes sur $A$. On considère alors la suite exacte de faisceaux sur $\mathbf{P}_{\Omega}^{3}$ :

$$
\mathcal{P}_{0} \otimes_{A} \Lambda \stackrel{w_{\lambda}}{\longrightarrow} \mathcal{N}_{0} \otimes_{A} \Lambda \rightarrow \mathcal{E}_{\Lambda} \rightarrow 0 .
$$

En vertu de 2.2 appliqué aux localisés de $A[\lambda]$, on voit que $w_{\lambda}$ est injectif et que son conoyau est plat, donc définit une famille de courbes $\mathcal{C}$ paramétrée par $\Omega$, dont les sections correspondant à $\lambda=0$ et $\lambda=1$ sont respectivement $\mathcal{C}_{0}$ et $\mathcal{C}_{1}$, comme annoncé. Comme les familles $\mathcal{C}_{a}$ pour $a \in A_{\Omega}$ ont toutes pour résolution $0 \rightarrow \mathcal{P}_{0} \rightarrow \mathcal{N}_{0} \rightarrow \mathcal{J}_{\mathcal{C}_{a}}(h) \rightarrow 0$, on voit que la déformation est à cohomologie uniforme.

Corollaire 2.10 (Propriété de Lazarsfeld-Rao). Soit $\mathcal{C}_{0}$ une famille minimale de courbes et $\mathcal{C}$ une famille de courbes de la classe de biliaison de $\mathcal{C}_{0}$. Alors, il existe un entier $m \geq 0$ et une suite de courbes $\mathcal{C}_{0}, \mathcal{C}_{1}, \ldots, \mathcal{C}_{m}$ telle que $\mathcal{C}_{i+1}$ s'obtienne à partir de $\mathcal{C}_{i}$ par une biliaison élémentaire (cf. [HMDP1]) et $\mathcal{C}$ à partir de $\mathcal{C}_{m}$ par une déformation à cohomologie uniforme.

Démonstration. La démonstration originelle de Lazarsfeld et Rao (cf. [MDP1] IV 5.1) s'applique presque sans changement. Elle repose sur le corollaire 2.9, la seule différence étant qu'on doit trouver une famille plate de surfaces (au lieu d'une seule surface) pour effectuer une biliaison. Il suffit pour cela de faire le raisonnement habituel au point fermé.

Remarque 2.11. Si le corps $k(t)$ est fini, les assertions d'existence de 2.5, 2.6, 2.9 et 2.10 restent vraies à condition de remplacer éventuellement $A$ par un anneau local $B$ fini et étale sur $A$.

\section{Exemples.}

a) Un algorithme de calcul.

Soit $A$ un anneau local noethérien, $T=\operatorname{Spec} A$ et $t$ le point fermé de $T$.

Considérons la situation suivante: On se donne deux $R_{A}$-modules libres gradués $L_{1}$ et $L_{2}$ et un homomorphisme de degré $0, s: L_{2} \rightarrow L_{1}$, donné par une matrice à coefficients homogènes dans $R_{A}$ que l'on note encore $s$. Soit $\widetilde{s}: \mathcal{L}_{2} \rightarrow \mathcal{L}_{1}$ la flèche de faisceaux associée à $s$. On pose $\mathcal{N}=\operatorname{Im} \widetilde{s}$ et $\mathcal{K}=$ Coker $\widetilde{s} ;$ on a donc la suite exacte $(*): 0 \rightarrow \mathcal{N} \rightarrow \mathcal{L}_{1} \rightarrow \mathcal{K} \rightarrow 0$ et on fait les deux hypothèses suivantes:

1) $\mathcal{K}$ est localement libre. Cette condition se traduit en termes matriciels: si $r$ est le rang de $s_{t}: L_{2, t} \rightarrow L_{1, t}$ (c'est-à-dire la dimension d'un plus grand mineur non nul de la matrice $s_{t}$ ) $\mathcal{K}$ est localement libre si et seulement si les $r$-mineurs de $s_{t}$ définissent le vide dans $\mathbf{P}_{k(t)}^{3}$. 
2) la flèche $L_{2} \rightarrow N=H_{*}^{0} \mathcal{N}$ induite par $s$ est surjective.

On notera que tout faisceau localement libre $\mathcal{N}$ sur $\mathbf{P}_{A}^{3}$ peut s'obtenir de cette manière : pour obtenir $L_{1}$ il suffit de considérer le dual $\mathcal{N}^{\vee}$ de $\mathcal{N}$ et de prendre une présentation libre $L_{1}^{\vee} \rightarrow H_{*}^{0} \mathcal{N}^{\vee} \rightarrow 0$ et pour obtenir $L_{2}$ de prendre une présentation libre $L_{2} \rightarrow N=H_{*}^{0} \mathcal{N} \rightarrow 0$.

Nous montrerons, dans [HMDP3], comment obtenir systématiquement des faisceaux $\mathcal{N}$ dits triadiques, munis de suites exactes

$$
0 \rightarrow \mathcal{N} \rightarrow \mathcal{L}_{1} \rightarrow \mathcal{L}_{0} \rightarrow \mathcal{L}_{-1} \rightarrow 0,
$$

avec les $\mathcal{L}_{i}$ dissociés qui conduisent naturellement à de telles écritures.

On se propose de calculer les invariants $\alpha_{n}=\alpha_{n}(\mathcal{N}), \beta_{n}=\beta_{n}(\mathcal{N}), b_{0}=$ $b_{0}(\mathcal{N})$ et la fonction $q=q_{\mathcal{N}}($ cf. 2.4).

On pose $L_{2}=\bigoplus_{k \in \mathbf{Z}} R_{A}(-k)^{l_{2}(k)}$ et $L_{2, \leq n}=\bigoplus_{k \leq n} R_{A}(-k)^{l_{2}(k)}$ et on appelle $s_{n}$ la restriction de $s$ à $L_{2, \leq n}$ (ou la matrice correspondante) et $s_{n, t}$ sa valeur au point fermé de $T$. On a alors les résultats suivants:

Proposition 3.1. Soit $n \in \mathbf{Z}$.

1) On a $\alpha_{n}=\operatorname{rang} s_{n, t}$, de sorte que $\alpha_{n}$ est le plus grand entier $\alpha$ tel que $s_{n, t}$ ait un $\alpha$-mineur non nul.

2) L'entier $\beta_{n}$ est le plus grand entier $\beta$ tel que les $\beta$-mineurs de $s_{n, t}$ soient sans facteur commun dans $R$.

3) Un entier $n$ est $\leq b_{0}$ si et seulement si il vérifie:

a) $\alpha_{n}=\beta_{n}$,

b) le sous-R-module $F$ de $L_{1, t}$ engendré par les colonnes de la matrice $s_{n, t}$ est libre de rang $\alpha_{n}$.

En particulier, si inf $L_{2}$ est le plus petit entier $n$ tel que $l_{2}(n)$ soit non nul, on a $b_{0} \geq \inf L_{2}-1$.

Démonstration. Considérons $\sigma_{N^{\prime}, n}: \mathcal{O}_{\mathbf{P}}(n) \otimes_{k} N_{n}^{\prime} \rightarrow \mathcal{N}_{t}$. Il s'agit de déterminer le rang de $\sigma_{N^{\prime}, n}$ aux points de codimension $\leq 1$ de $\mathbf{P}^{3}$. Comme $L_{2, t} \rightarrow N^{\prime}$ est surjectif il revient au même de calculer le rang de $s_{n, t}^{\prime}$ où $s_{n, t}^{\prime}:\left(\mathcal{L}_{2, \leq n}\right)_{t} \rightarrow \mathcal{N}_{t}$ est induite par $s_{n, t}$. Comme la suite $(*)$ est localement scindée, il revient encore au même de calculer le rang de $\widetilde{s_{n, t}}$ où $\widetilde{s_{n, t}}:\left(\mathcal{L}_{2, \leq n}\right)_{t} \rightarrow \mathcal{L}_{1, t}$ a pour matrice $s_{n, t}$ et on en déduit aussitôt 1 ) et $2)$.

Pour l'assertion 3), supposons d'abord $n \leq b_{0}$. On a alors $\alpha_{n}=\beta_{n}$ (cf. 1.12). De plus, en vertu de 1.14, le sous-module $N_{\leq n}^{\prime}$ de $H^{0} \mathcal{N}_{t}$ est libre de rang $\alpha_{n}$. Mais, comme $\left(L_{2, \leq n}\right)_{t} \rightarrow N_{\leq n}^{\prime}$ est surjectif et $\mathcal{N}_{t} \rightarrow \mathcal{L}_{1, t}$ injectif, $N_{<n}^{\prime}$ n'est autre que $F$.

Réciproquement, si on a les conditions a) et b) ci-dessus, on considère le faisceau $\mathcal{L}=\mathcal{L}_{2, \leq n}$. Comme $F$ est libre de rang $\alpha_{n}$, on peut, quitte à faire un changement de base et à supprimer éventuellement des colonnes de $s$, supposer que $\mathcal{L}$ est dissocié de rang $\alpha_{n}$. La flèche $s_{n, t}$ est alors injective et, 
comme on a $\alpha_{n}=\beta_{n}$, son conoyau est sans torsion (cf. [MDP3] II 2.2) et on conclut par 1.14 .

b) Exemples.

Nous donnons quelques exemples très simples d'application de la méthode ci-dessus. Le lecteur qui souhaiterait avoir plus d'exemples, ou des exemples plus complexes, ou encore comprendre comment ces exemples sont construits, ou enfin quelles sont les relations de ces exemples avec les spécialisations dans le schéma de Hilbert, se reportera à [HMDP3].

Dans tout ce qui suit on suppose que $A$ est une $k$-algèbre qui est un anneau de valuation discrète d'uniformisante $a$.

Les exemples ci-dessous sont tous construits de la même façon: on prend une suite exacte de $R=k[X, Y, Z, T]$-modules libres gradués: $M_{2} \stackrel{\sigma_{2}}{\longrightarrow} M_{1} \stackrel{\sigma_{1}}{\longrightarrow}$ $M_{0}$ telle que le faisceau Coker $\widetilde{\sigma}_{1}$ soit localement libre. On obtient par exemple une telle suite en prenant trois termes consécutifs dans la résolution libre d'un $R$-module de longueur finie. On considère alors $s: L_{2}=\left(M_{1} \otimes_{k}\right.$ A) $\oplus\left(M_{2} \otimes_{k} A\right) \rightarrow L_{1}=\left(M_{0} \otimes_{k} A\right) \oplus\left(M_{1} \otimes_{k} A\right)$ donné par la matrice

$$
s=\left(\begin{array}{cc}
\sigma_{1} & 0 \\
a I & \sigma_{2}
\end{array}\right) .
$$

On vérifie que Coker $\widetilde{s}$ est localement libre, de sorte qu'on peut appliquer 3.1 à $\mathcal{N}=\operatorname{Im} \widetilde{s}\left(L_{2} \rightarrow N\right.$ est surjective $)$.

Dans les Exemples 3.2 et 3.3 on part de la résolution du $R$-module $k=$ $R /(X, Y, Z, T)$ donnée par le complexe de Koszul associé à la suite régulière $U=(X, Y, Z, T)$ :

$$
\begin{gathered}
0 \rightarrow R(-4) \rightarrow R(-3)^{4} \stackrel{V^{\prime}}{\longrightarrow} R(-2)^{6} \stackrel{V}{\longrightarrow} R(-1)^{4} \stackrel{U}{\longrightarrow} R \rightarrow k \rightarrow 0, \\
V=\left(\begin{array}{cccccc}
Y & Z & T & 0 & 0 & 0 \\
-X & 0 & 0 & Z & T & 0 \\
0 & -X & 0 & -Y & 0 & T \\
0 & 0 & -X & 0 & -Y & -Z
\end{array}\right) \quad \text { et } V^{\prime}=\left(\begin{array}{cccc}
0 & 0 & -T & Z \\
0 & T & 0 & -Y \\
0 & -Z & Y & 0 \\
-T & 0 & 0 & X \\
Z & 0 & -X & 0 \\
-Y & X & 0 & 0
\end{array}\right) .
\end{gathered}
$$

Exemple 3.2. On considère la matrice $s: R_{A}(-1)^{4} \oplus R_{A}(-2)^{6} \rightarrow R_{A} \oplus$ $R_{A}(-1)^{4}$ avec $s=\left(\begin{array}{cc}U & 0 \\ a I_{4} & V\end{array}\right)$ où $I_{4}$ est la matrice identité d'ordre 4. Au point fermé (i.e., pour $a=0$ ) il ne reste dans $s_{1}$ que la matrice ligne $U$ ce qui montre, en vertu de 3.1, qu'on a $\alpha_{1}=\beta_{1}=1$. Comme les colonnes de cette matrice engendrent l'idéal $m=(X, Y, Z, T)$ qui n'est pas libre sur $R$ on a $b_{0}=0$. En degré 2 on vérifie aussitôt qu'on a $\alpha_{2}=\beta_{2}=4$. On en déduit que la seule valeur non nulle de la fonction $q$ est $q(2)=3$ et, en vertu 
de 2.6, qu'on a une suite exacte

$$
0 \rightarrow \mathcal{O}_{\mathbf{P}_{A}}(-2)^{3} \stackrel{u}{\longrightarrow} \mathcal{N} \rightarrow \mathcal{J}_{\mathcal{C}}(2) \rightarrow 0
$$

où $\mathcal{C}$ est une famille de courbes, plate sur $A$ et à spécialité constante. Ces courbes sont de degré 6 et de genre 3 . Pour $u$ général, la courbe générique $C$ de la famille $\mathcal{C}$ est une courbe arithmétiquement de Cohen-Macaulay (ACM) lisse tandis que la courbe spéciale $C_{0}$ est une courbe lisse de bidegré $(2,4)$ tracée sur une quadrique lisse. Cette courbe est dans la classe de biliaison de deux droites disjointes et son module de Rao est concentré en degré 2 .

Exemple 3.3. On considère la matrice $s: R_{A}(-2)^{6} \oplus R_{A}(-3)^{4} \rightarrow R_{A}(-1)^{4}$ $\oplus R_{A}(-2)^{6}: s=\left(\begin{array}{cc}V & 0 \\ a I_{4} & V^{\prime}\end{array}\right)$ et le faisceau $\mathcal{N}$ associé. On a $\alpha_{2}=\beta_{2}=3$ et $b_{0}=1$, puis $a_{3}=\beta_{3}=6$ donc la fonction $q$ vérifie $q(2)=2$ et $q(3)=3$. La famille minimale de courbes associée à $\mathcal{N}$ est encore une famille de courbes de degré 6 et genre 3 comme dans l'exemple 3.2, mais, cette fois-ci, il s'agit d'une famille à postulation constante. Pour un homomorphisme général, la courbe générique est une courbe ACM lisse et la courbe spéciale est la réunion d'une quartique plane et de deux droites qui coupent chacune la quartique transversalement en un point. Cette courbe est dans la classe de biliaison de deux droites disjointes et son module de Rao a un unique terme non nul en degré 1 (et non plus en degré 2 comme dans l'exemple 3.2). Pour une étude plus approfondie du schéma de Hilbert $H_{6,3}$ cf. [AA].

Exemple 3.4. Nous donnons ici un exemple avec une partie "obligatoire" au sens de 1.6 c'est-à-dire un exemple dans lequel il existe un entier $n \leq b_{0}$ avec $q(n) \neq 0$.

On considère un module $M$ de longueur finie concentré en degrés 0 et 1 et de dimensions respectives 2 et 7 . On suppose ce module générique et il admet alors une résolution de la forme suivante (cf. [MDP2] IV 1.2)

$$
\begin{aligned}
& \cdots \rightarrow R(-3)^{34} \stackrel{\sigma_{2}}{\longrightarrow} R(-1) \oplus R(-2)^{16} \stackrel{\sigma_{1}}{\longrightarrow} R^{2} \rightarrow M \rightarrow 0 \quad \text { avec } \\
& \sigma_{1}=\left(\begin{array}{ccccccccccccccccc}
X & Y^{2} & Z^{2} & T^{2} & Y Z & Y T & Z T & 0 & 0 & 0 & 0 & 0 & 0 & 0 & 0 & 0 & 0 \\
-Y & 0 & 0 & 0 & 0 & 0 & 0 & X^{2} & Y^{2} & Z^{2} & T^{2} & X Y & X Z & X T & Y Z & Y T & Z T
\end{array}\right) .
\end{aligned}
$$

On considère alors $s: R_{A}(-1) \oplus R_{A}(-2)^{16} \oplus R_{A}(-3)^{34} \stackrel{s}{\longrightarrow} R_{A}^{2} \oplus R_{A}(-1) \oplus$ $R_{A}(-2)^{16}$

$$
\text { donné par } \quad s=\left(\begin{array}{cc}
\sigma_{1} & 0 \\
a I_{17} & \sigma_{2}
\end{array}\right) .
$$

La matrice $s$ admet une seule colonne en degré $\leq 1$, dont la transposée est la ligne $(X,-Y,-a, 0, \cdots, 0)$. On en déduit aussitôt en utilisant 3.1 qu'on a $\alpha_{1}=\beta_{1}=1$ et $b_{0} \geq 1$ et donc, par définition de la fonction $q, q(1)=1$. On a aussi $q(3)=15$ et la famille minimale est en degré 120 et genre 1001 avec une courbe générique ACM. 


\section{References}

[AA] S. Aït-Amrane, Sur le schéma de Hilbert des courbes gauches de degré d et genre $g=(d-3)(d-4) / 2$, thése, Université Paris-Sud Orsay, 1998.

[EGA] A. Grothendieck et J. Dieudonné, Éléments de géométrie algébrique III, Publ. Math. IHES, 11 (1961) et IV, Publ. Math. IHES, 28 (1966).

[AG] R. Hartshorne, Algebraic geometry, Graduate texts in Mathematics, 52, Springer Verlag, 1977.

[H] _ Coherent functors, Advances in Math., 140 (1998), 44-94.

[HMDP1] R. Hartshorne, M. Martin-Deschamps et D. Perrin, Un théorème de Rao pour les familles de courbes gauches, J. Pure and Applied Algebra, à paraître.

[HMDP3] _ Triades et familles de courbes gauches, Math. Ann., 315 (1999), 397468.

[K] S.L. Kleiman, Geometry on Grassmannians and applications to splitting bundles and smoothing cycles, Publ. Math. IHES, 36 (1969), 281-297.

[LR] R. Lazarsfeld et A.P. Rao, Linkage of general curves of large degree, Lecture notes, 997, Springer Verlag, (1983), 267-289.

[MDP1] M. Martin-Deschamps et D. Perrin, Sur la classification des courbes gauches, Astérisque, Vol. 184-185, 1990.

[MDP2] Courbes gauches et Modules de Rao, J. Reine Angew. Math., 439 (1993), 103-145.

[MDP3] _ Construction de courbes lisses: un théorème à la Bertini, rapport de recherche du LMENS 92-22, 1992.

[MDP4] _ Quand un morphisme de fibrés dégénère-t-il le long d'une courbe lisse, in 'Algebraic Geometry', Marcel Dekker, Inc., Lecture Notes in Pure and Applied Mathematics, 200 (1998), 119-167.

[R] A.P. Rao, Liaison equivalence classes, Math. Ann., 258 (1981), 169-173.

[S1] E. Schlesinger, Vms fundamentals, unpublished manuscript, Berkeley, 1992.

Received January 10, 1998.

University OF CALifornia

BERKELEy, CA 94720-3840

E-mail address: robin@math.berkeley.edu

UNIVERSITY OF CALIFORNIA

Berkeley, CA 94720-3840

UNIVERSiTy OF CALIFORNIA

Berkeley, CA 94720-3840 